Thorax (1948), 3, 79.

\title{
LIVER BIOPSY IN SARCOIDOSIS
}

\author{
BY \\ J. G. SCADDING AND SHEILA SHERLOCK* \\ From the Department of Medicine, Postgraduate Medical School of London, and the Institute of Diseases \\ of the Chest, Brompton Hospital, London
}

No agreement has been reached about the aetiology of the syndrome known as sarcoidosis, Besnier-Boeck-Schaumann disease, lymphogranulomatosis benigna, or non-caseating tuberculosis; and even those who hold decided opinions on this subject admit their inability to demonstrate the agent which they consider responsible. Hence any satisfactory definition of the group of cases to be included under this heading includes a description of the characteristic histological changes, and the most secure basis for a diagnosis of sarcoidosis in the individual case is the demonstration of these changes in affected tissues. If superficial lymphnodes are enlarged, they provide suitable biopsy material ; but in some of the more obscure cases, where diagnosis is often of practical importance, these nodes are not involved.

In 1943 van Beek and Haex first drew attention to the possible value of aspiration liver biopsy in providing favourable material for the histological diagnosis of sarcoidosis. They reported that in two out of four cases presenting features of this syndrome they had been able to find characteristic changes in the material obtained by aspiration liver biopsy, although in neither of them had there been clinical evidence of hepatic involvement. Subsequently van Buchem (1946) published a further. series of fourteen cases in which liver biopsy was performed, with positive results in all. In view of the controversy about the relation between sarcoidosis and tuberculosis van Buchem also carried out liver biopsy in nine cases of active pulmonary tuberculosis. In none were specific changes found in the material examined.

The present paper reports three cases presenting clinical manifestations of sarcoidosis, in which characteristic changes were found in liver tissue obtained by aspiration biopsy, and a fourth in which similar changes were an incidental finding in a liver biopsy from a patient showing no other evidence of this syndrome.

\section{CAse Reports}

Case 1.-A single woman, aged 21 , employed as a shorthand typist, had had no serious illnesses and felt quite fit, leading an active life, when in November, 1945 , she was shown by mass radiography to have a dense miliary infiltration of both lungs. This infiltration (Plate XVIá) has changed very little, except that recently (January, 1948) it has become rather denser.

Clinical findings.-The general condition was very good, and the patient was afebrile during two periods of hospital observation. No abnormal physical signs have ever been found, except that when she first came under observation lymph-nodes just above the inner ends of the clavicles and in the right epitrochlear region were enlarged to about $1 \mathrm{~cm}$. diameter. The right supraclavicular node was removed for biopsy in March, 1946, and showed characteristic changes of sarcoidosis (Plate XVIb). The gland structure was mostly destroyed, but some lymphoid tissue and much of the peripheral sinus remained. The greater part of the gland was occupied by tubercle-like collections of epithelioid cells, with a very few small giant cells but no caseation. The intervening tissue consisted of dense, not very cellular collagen. ZiehlNielsen and fluorescent stains showed no acid-fast bacilli.

Liver biopsy (Plate XVII).-This was performed in March, 1946. One minute sarcoid lesion was found in one section only after serial section of the whole biopsy specimen. The lobular pattern of the liver, the sinusoids, and portal tracts was normal. The liver cells contained an occasional fat droplet, but less fat than was seen in Case 3 ; their glycogen content was normal. The lesion was minute, its diameter being about five times that of a liver cell. In the section it consisted of about twelve epithelioid cells with a few scattered lymphocytes, and was so well circumscribed by a few fibroblasts that definition from the surrounding liver was easy. There were no giant cells. Staining for acid-fast bacilli could not be done as no further sections were available.

Mantoux reaction.-This was persistently negative to $10 \mathrm{mg}$. old tuberculin.

Radiographs of hands and feet.-These showed no bone changes. 
Plasma proteins (January, 1948). - The total was 7.2 g., albumin 3.8 g., globulin 3.4 g. per $100 \mathrm{mr}$.

Progress.-During two years' observation there has been little change in the findings. In January, 1948, however, the patient was found to have lost some weight, the sedimentation rate had risen to $55 \mathrm{~mm}$. (Westergren), and the pulmonary infiltration looked rather denser. No superficial lymph-nodes were palpable at this time, except the previously much enlarged right epitrochlear, which had diminished in size and was only just palpable. Radiographs of the hands and feet showed that oval areas of translucency had appeared in the head of the proximal phalanx of the left index finger, in the base of the proximal phalanx of the left middle finger, in the heads of both middle metatarsals, and in the terminal phalanx of the left great toe.

Case 2.-A railway clerk, aged 24, complained of dimness of vision in the right eye in May, 1945, and was found to have iridocyclitis. A radiograph of the chest was normal. The vision returned to normal in about one month. In May, 1946, the vision in both eyes became dim, and he was treated without benefit for iridocyclitis in both eyes with injections of T.A.B. vaccine and with penicillin. $A$ radiograph of the chest at this time showed enlarged right hilar glands; the hands showed no bone changes.

He was referred to the Brompton Hospital in February, 1947. The general condition was excellent. There was bilateral kerato-iridocyclitis with vitreous opacities, more severe in the left eye; the fundi were indistinct, but showed no gross choroidal lesions. There were no enlarged superficial lymphnodes. The spleen was easily palpable, firm in consistence, and coming down about $4 \mathrm{~cm}$. below the costal margin on inspiration. There were no other abnormal physical signs.

Radiograph of chest (Plate XVIII a).-This showed enlarged glands at the right hilum.

Sputum.-No acid-fast bacilli were found on examination by concentration method.

Mantoux reaction.-This was moderately positive to $0.005 \mathrm{mg}$. purified protein derivative.

Plasma proteins.-The total was 6.6 g., albumin 4.7 g., globulin 1.9 g. per $100 \mathrm{ml}$.

Liver biopsy (Plate XVIII b).-Biopsy showed a normal lobular pattern; the cells were normal, with their full complement of glycogen; there was no increase in cells in the sinusoids; the portal tracts were normal. Two sarcoid lesions were seen. The larger was mid-zonal and well circumscribed; its size was about one-third of that of a liver lobule; it was composed of central epithelioid cells with pale cytoplasm and pale open nuclei, surrounded by smaller cells with dark nuclei. No giant cells were seen. There were a few fibroblasts at the periphery. No acid-fast bacilli were seen in appropriately stained sections.
The smaller lesion was a minute circumscribed cluster of dark cells and an occasional fibroblast, with very few of the larger pale cells.

Progress.-He was treated with calciferol 150,000 units daily from April 19 to May 21, 1947, when the calciferol treatment was stopped on account of abdominal discomfort, nausea, and loss of weight, with a rise of serum calcium to $14.6 \mathrm{mg}$. per $100 \mathrm{ml}$. This systemic reaction to calciferol gradually subsided; there was no subsequent change in the size of the hilar lymph-nodes or the spleen, but the ocular condition became much quieter.

In December, 1947, the eyes showed no active lesions, though the vision had been reduced by residual opacities in the media. The spleen and hilar lymph-nodes were unchanged in size, but the patient felt quite well and was working.

Case 3.-A housewife, aged 40, complained of gradually progressive dyspnoea on exertion, precordial pain, cough with scanty mucoid sputum, and slight loss of weight for eighteen months.

She was admitted to the Brompton Hospital on May 7, 1947. The general condition was good; she was of lean type, with a high colour. There was a small group of enlarged lymph-nodes in the left axilla, but no other superficial lymph-nodes were palpable. There were persistent fine rales all over both lungs. The spleen was palpable, of firm consistence, and coming down about $2 \mathrm{~cm}$. below the costal margin on deep inspiration.

Radiograph of the chest.-Curious fine reticular markings showed in the upper two-thirds of both lung fields; there was no evidence of hilar lymphnode enlargement.

Radiograph of the hands.-Radiograph of the hands showed no bone changes.

Sedimentation rate (Westergren). - This was $10 \mathrm{~mm}$. in first hour.

Mantoux reaction.-This was negative to $1 \mathrm{mg}$. old tuberculin.

Vital capacity.-Vital capacity was $1,300 \mathrm{ml}$.

Plasma proteins.-The total was 6.9 g., albumin 5.0 g. per $100 \mathrm{ml}$.

Biopsy of axillary lymph-node.-Biopsy showed multiple rounded collections of endothelial cells with a very few round cells (Plate XIXa). No tubercle bacilli were found in appropriately stained sections.

Liver biopsy (Plate XIXb).-The lobular pattern was normal. The liver cells showed slight fatty change, maximal periportally, but a normal glycogen content ; sinusoids and portal tracts were normal. One sarcoid lesion was seen. It was about one-quarter of the size of a lobule and adjoined a portal tract. It consisted of a pale pink acellular homogeneous centre containing mature connective tissue. The extreme centre showed a few epithelioid cells and younger fibroblasts. Dark round cells occupied a narrow zone 
at the periphery ; there were no giant cells. No acidfast bacilli were found.

These appearances suggested a later, less active stage of the process than was seen in Case 2. This correlated well with the clinical picture, since the radiograph of the lung suggested a fine fibrosis consequent on the healing of sarcoid lesions.

The subsequent progress of the patient has borne out this view, since there has been no appreciable change in her condition.

Case 4.-A girl aged 15 was brought into Hammersmith Hospital on May 20, 1946, with a history of having drunk between 1 and $1 \frac{1}{2} \mathrm{oz}$. ( 30 and $45 \mathrm{ml}$.) of carbon tetrachloride. She vomited about two hours later, and was admitted to hospital ten hours after the incident. Her general condition was very good, and there were no abnormal physical signs except some epigastric tenderness. The stomach was washed out and traces of carbon tetrachloride were found to be still present. She developed no more serious symptoms and made an uneventful recovery.

The previous personal history and the family history contained nothing relevant.

Blood count.-The blood count was normal.

Urine.-The urine contained no abnormal constituent.

Erythrocyte sedimentation rate.-This was $2 \mathrm{~mm}$. (Westergren).

Liver biopsy (Plate XX).-Biopsy showed a normal lobular pattern, with occasional hydropic degeneration and fatty change in the cells at the centres of the lobules. Two sarcoid-like lesions were seen. The larger occupied an area in the section about twothirds of that of a liver lobule. At its centre there was a little amorphous debris, but no definite caseation; outside this was a middle zone of epithelioid cells; and peripherally a zone of epithelioid cells, lymphocytes, and a few giant cells. The smaller lesion consisted, like that found in Case 1, entirely of epithelioid cells. The larger lesion seemed to have developed in a periportal region, and a few bile ducts are buried in one part of its peripheral zone.

A second biopsy obtained twelve days later showed the fatty changes to have cleared, and no sarcoid lesions were found in this specimen.

Mantoux reaction.-This was positive to $0.01 \mathrm{mg}$. old tuberculin.

Sternal puncture.-Sternal puncture showed no tubercles or sarcoid-like lesions and no acid-fast bacilli.

Radiographs.-Radiographs of the chest and of the hands showed no abnormality.

Progress.-This patient has been uncooperative in follow-up. She was in hospital, however, in June, 1947. for an unrelated gynaecological complaint, and was found to have developed no clinical manifestation of sarcoidosis.

\section{Discussion}

Autopsy reports of sarcoid lesions in the liver.The number of published reports of autopsies in cases of sarcoidosis is small ; Pinner (1937) collected seventeen and added one of his own, and Rubin and Pinner (1944) added twenty-five more in a comprehensive review of the literature. Of the total of forty-three cases so reviewed, seventeen showed lesions in the liver, although none had shown clinical evidence of liver involvement except slight enlargement in a few. Since the date of this review, Granström and others (1946) have published an autopsy report on a case of uveoparotid fever, in which sparse submiliary tubercles without caseation were found in the liver.

Site of the lesions in the liver.-Sarcoid lesions found in the liver at autopsy have been stated to be situated most commonly in the vicinity of the portal tracts and less commonly within the liver lobules. Thus Nickerson (1937), who found liver lesions in five cases out of six, stated that they "occurred in greatest numbers in the portal fibrous tissue, but were also sometimes found in the lobules in a mid-zonal position." Cotter (1939), in one case, described the arrangement of the lesions as "mainly periportal." Hollister and Harrell (1941), also reporting one case, found the lesions "in the inter-acinar connective tissue, most often in portal areas."

In biopsy specimens, van Beek and Haex (1943a and b) found the lesions in one case in the portal areas and in one mainly in the liver parenchyma ; van Buchem (1946) stated that " the foci are usually situated in the triangles of Kirnan and sometimes found spread about in the parenchyma." In our cases, the lesions found were equally distributed between the vicinity of the portal tracts and the mid-zones of the liver lobules.

Liver function in sarcoidosis.-Harrell and Fisher (1939) found an abnormal bilirubin tolerance test in four cases of sarcoidosis. Van Buchem (1946), however, found an abnormal oral galactose tolerance test and Takata-Ara reaction in only one of his fourteen cases. This patient showed the greatest liver involvement. In our liver biopsy sections, apart from minimal fatty change in two instances, the liver cells appeared normal. Moreover, the focal sarcoid lesions are very small and scattered. Detectable change in liver function would not be expected. Routine liver function tests will be of little value in the diagnosis of hepatic sarcoidosis.

The incidental finding of sarcoid lesions.-Of Nickerson's (1937) six cases in which sarcoid 

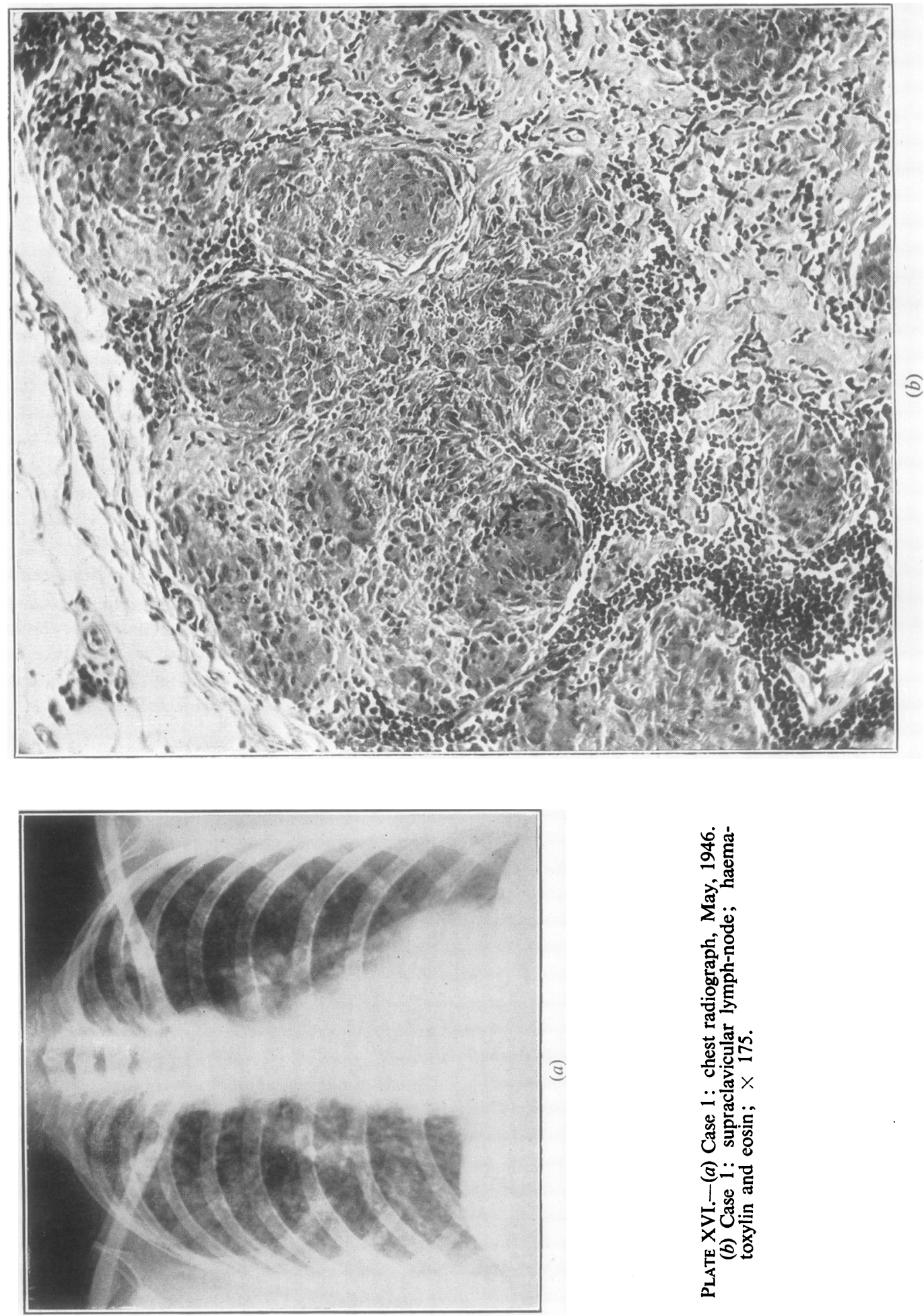


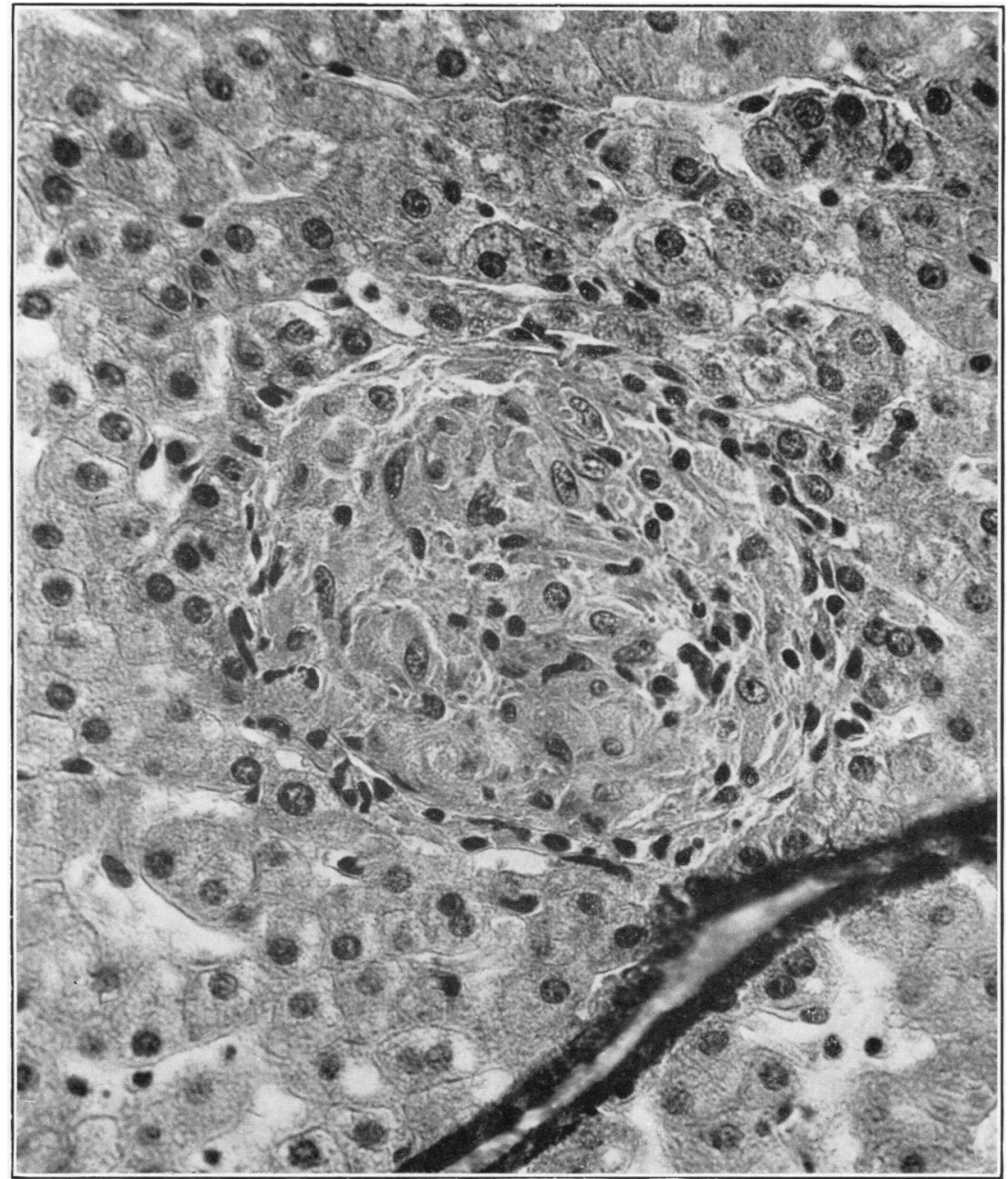

Plate XVII.-Case 1:' liver biopsy; haematoxylin and eosin; $\times 430$. 

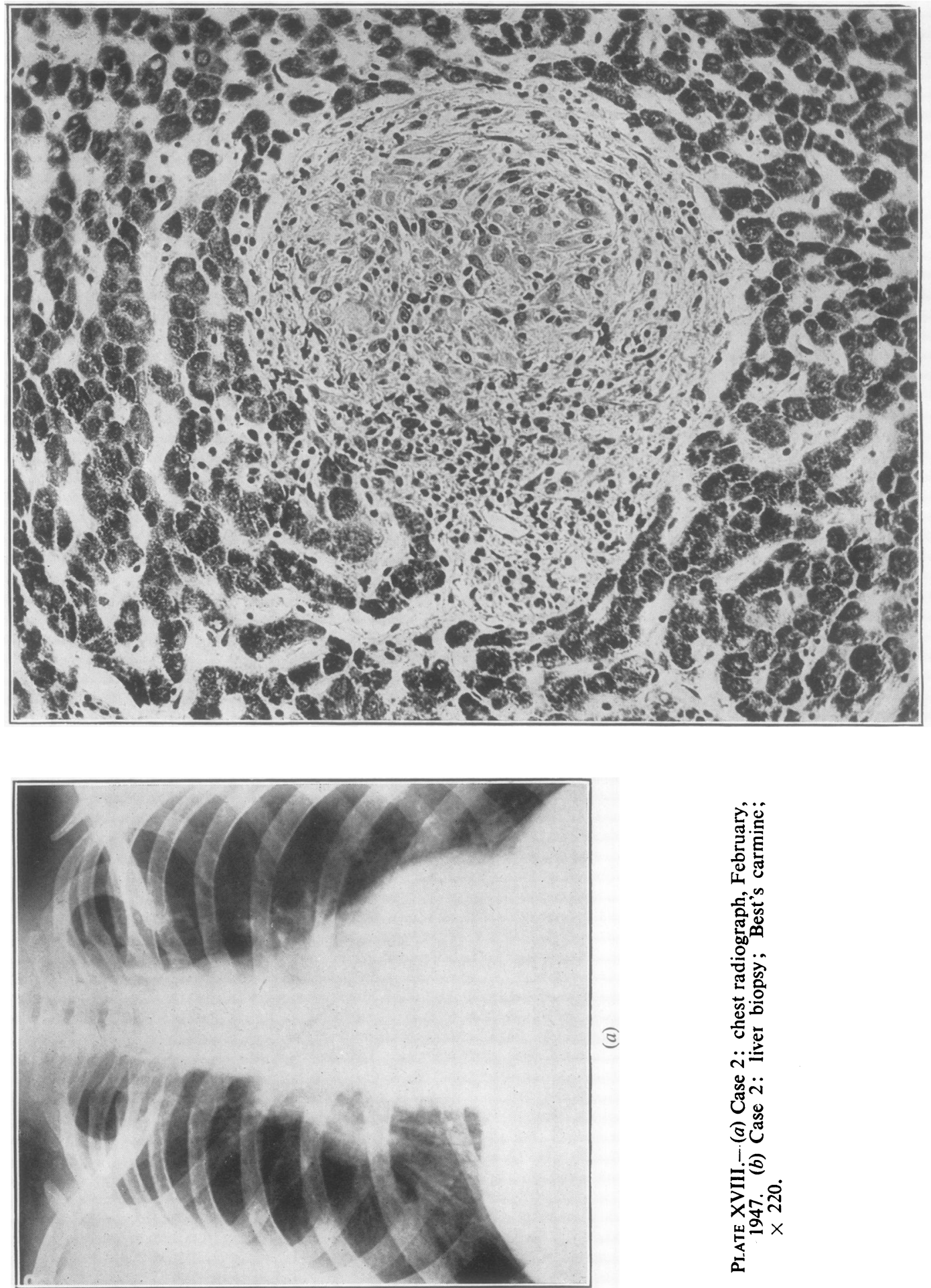


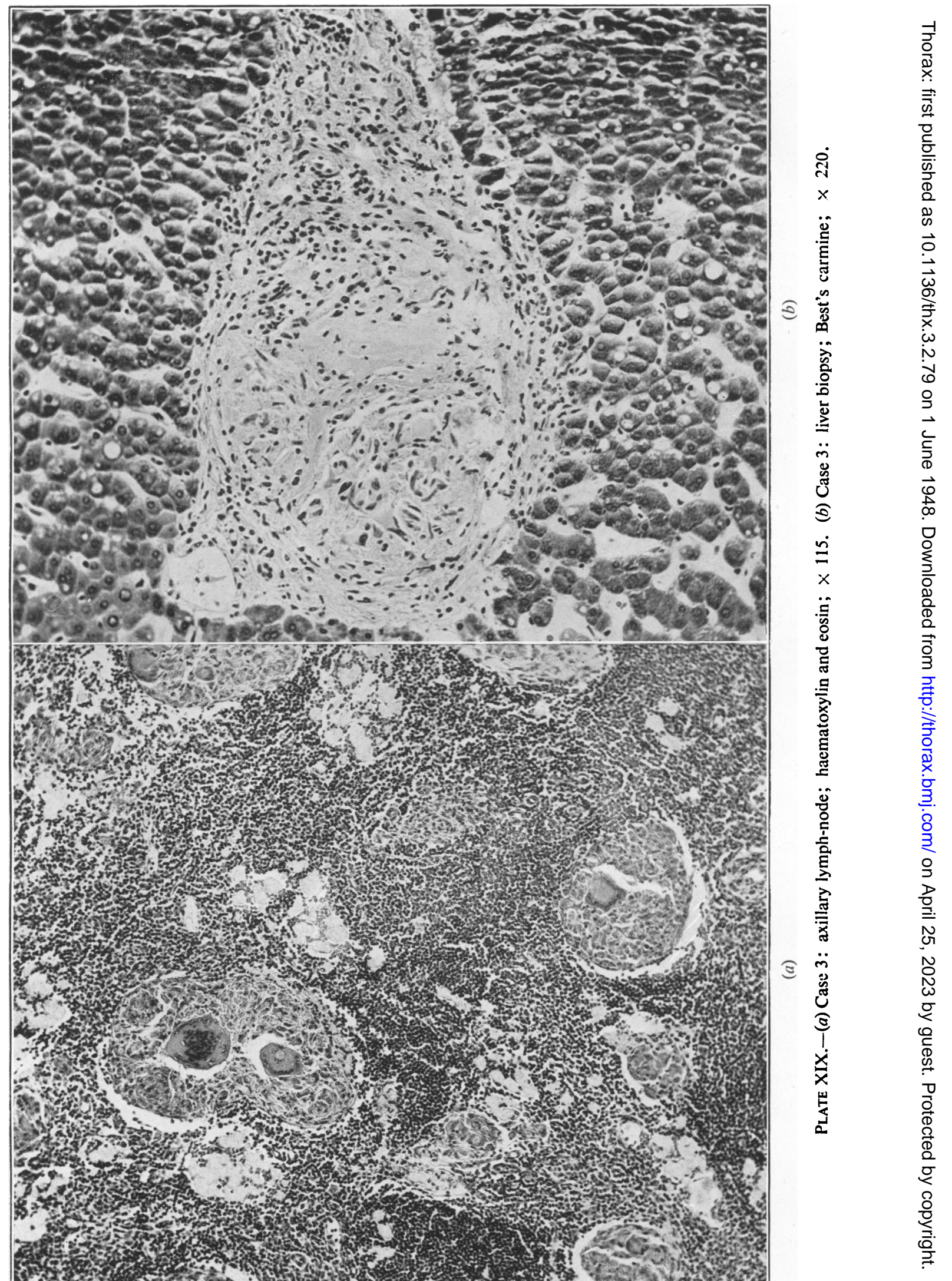




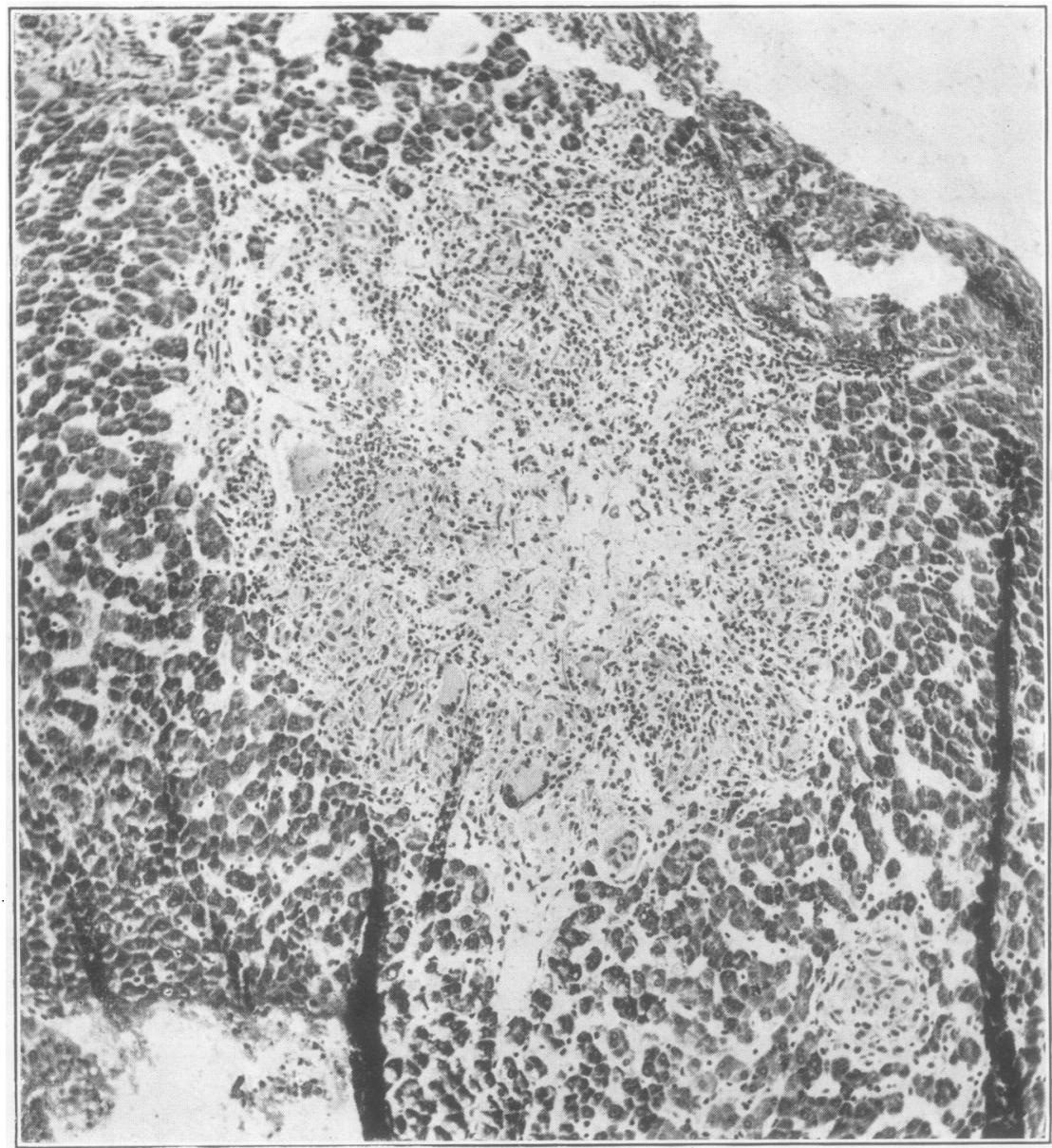

Plate XX.-Case 4: liver biopsy; Best's carmine; $\times 115$. 
lesions were found at autopsy, in only one was sarcoidosis the cause of death. In the remaining five, death was due to intracranial haemorrhage of various types (three cases), lobar pneumonia (two cases), and bronchopneumonia with heart failure (one case), and sarcoidosis had not been suspected clinically. In four of these five, lesions were present in the liver; if, therefore, sarcoidosis had been suspected during life, liver biopsy would have offered a good chance of obtaining histological proof of this diagnosis.

In 450 liver biopsies performed by one of us (S.S.), on patients suffering from a wide range of diseases, that in Case 4 is the first in which a sarcoid-like les:on has been an unexpected incidental finding. This lesion might be regarded as a single miliary tubercle of classical type, especially since the Mantoux reaction was positive to $0.01 \mathrm{mg}$. old tuberculin ; but the good general condition of the patient and the absence of other evidence of active tuberculosis, either at the time of the biopsy or developing later, is more in keeping with the view that it was of sarcoid type. Whether the distinction between tubercles of classical type and of sarcoid type is regarded as of kind or of degree depends, of course, upon the view adopted about the aetiology of sarcoidosis.

The value of liver biopsy in the diagnosis of sarcoidosis.-Cases 1, 2, and 3 were the only ones we saw during 1946 and 1947, in which the clinical aspects suggested a diagnosis of sarcoidosis, and in all of them lesions were found in the liver by aspiration biopsy. In two of them the diagnosis was also confirmed by biopsy of lymph-nodes, but in the third the liver biopsy alone gave histological proof of the diagnosis. The published experience of van Beek and Haex (1943a and b) and of van Buchem (1946) has already been quoted ; to summarize their results and ours, in twenty-one cases diagnosed clinically as sarcoidosis, sixteen showed typical lesions in aspiration biopsies of the liver. The lesions often being small and scattered, it is important to cut serially through the entire biopsy if the first section shows no lesion; this is well demonstrated by Case 1. Since liver biopsy by a proper technique and with precautions is a safe procedure (Sherlock, 1945), it forms a useful addition to routine methods in the diagnosis of this sometimes rather obscure syndrome.

\section{Summary}

Aspiration liver biopsies in three consecutive cases presenting clinical features suggesting a diagnosis of sarcoidosis showed typical non-caseating tubercles. A similar lesion was found in a liver biopsy from a patient presenting no other evidence of this syndrome.

We are indebted to Mr. E. V. Willmott for the photomicrographs, and to Mr. J. C. Griffin and Mr. J. R. Baker for the histological preparations of the liver biopsies.

\section{REFERENCES}

Cotter, E. F. (1939). Arch. intern. Med., 64, 286.

Granström, K. O., Gripwall, E., Kristofferson, C. E., and Lingdren, A. G. H. (1946). Acta med. scand., 126, 307.

Harrell, G. T., and Fisher, S. (1939). J. clin. Invest., $18,687$.

Hollister, W. F.. and Harrell, G. T. (1941). Arch. Path., 31, 178.

Nickerson, D. A. (1937). Arch. Path., 24, 19.

Pinner, M. (1937). Amer. Rev. Tuberc., 37, 690.

Rubin, E. H.. and Pinner, M. (1944). Amer. Rev. Tuberc., 49, 146.

Sherlock, S. (1945). Lancet, 2, 397.

van Beek, C., and Haex, A. J. C. (1943a). Ned. Tijdschr. Geneesk., 87, 1264.

van Beek, C., and Haex, A. J. C. (1943b). Acta med. scand., 113, 125.

van Buchem, F. S. P. (1946). Acta med. scand., 124, 168. 